\title{
Early diagnosis of Pearson syndrome in neonatal intensive care following rapid mitochondrial genome sequencing in tandem with exome sequencing
}

\author{
Lauren S. Akesson ${ }^{1,2,3} \cdot$ Stefanie Eggers ${ }^{1}$ - Clare J. Love ${ }^{1} \cdot$ Belinda Chong ${ }^{1}$ - Emma I. Krzesinski ${ }^{3,4}$. \\ Natasha J. Brown ${ }^{1,2} \cdot$ Tiong Y. Tan ${ }^{1,2}$. Christopher M. Richmond ${ }^{1} \cdot$ David R. Thorburn $\mathbb{1}^{1,2,5}$. \\ John Christodoulou $\mathbb{1}^{1,2,5,6} \cdot$ Matthew F. Hunter ${ }^{3,4} \cdot$ Sebastian Lunke $\mathbb{1}^{1,6,7} \cdot$ Zornitza Stark $^{1,2,6}$
}

Received: 16 March 2019 / Revised: 23 June 2019 / Accepted: 3 July 2019 / Published online: 29 July 2019

(c) The Author(s), under exclusive licence to European Society of Human Genetics 2019

\begin{abstract}
Rapid genomic testing is a valuable new diagnostic tool for acutely unwell infants, however exome sequencing does not deliver clinical-grade mitochondrial genome sequencing and may fail to diagnose mitochondrial disorders caused by mitochondrial DNA (mtDNA) variants. Rapid mitochondrial genome sequencing and analysis are not routinely available in rapid genomic diagnosis programmes. We present two critically ill neonates with transfusion-dependent anaemia and persistent lactic acidosis who underwent rapid mitochondrial genome sequencing in tandem with exome sequencing as part of an exome sequencing-based rapid genomic diagnosis programme. No diagnostic variants were identified on examination of the nuclear exome data for either infant. Mitochondrial genome sequencing identified a large mtDNA deletion in both infants, diagnosing Pearson syndrome within 74 and 55 h, respectively. Early diagnosis in the third week of life allowed the avoidance of a range of other investigations and appropriate treatment planning. Rapid mitochondrial genome analysis provides additional diagnostic and clinical utility and should be considered as an adjunct to exome sequencing in rapid genomic diagnosis programmes.
\end{abstract}

These authors contributed equally to this manuscript as joint first authors: Lauren S. Akesson, Stefanie Eggers

These authors contributed equally to this manuscript as joint senior authors: Sebastian Lunke, Zornitza Stark

Zornitza Stark

Zornitza.Stark@vcgs.org.au

1 Victorian Clinical Genetics Services, Murdoch Children's Research Institute, Melbourne, VIC, Australia

2 Department of Paediatrics, University of Melbourne, Melbourne, VIC, Australia

3 Monash Genetics, Monash Medical Centre, Melbourne, VIC, Australia

4 Department of Paediatrics, Monash University, Melbourne, VIC, Australia

5 Brain and Mitochondrial Research Group, Murdoch Children's Research Institute, Melbourne, VIC, Australia

6 Australian Genomics Health Alliance, Melbourne, VIC, Australia

7 Department of Clinical Pathology, University of Melbourne, Melbourne, VIC, Australia

\section{Introduction}

Acutely unwell infants often present with undifferentiated clinical features and a broad differential diagnosis, which may include monogenic and mitochondrial disorders [1-3]. Genomic testing, including exome and genome sequencing (ES/GS), can assist the diagnostic assessment, with the potential to target therapy, clarify prognosis, avoid unnecessary or invasive investigations, and decrease health costs $[4,5]$. Rapid genomic testing can deliver a molecular diagnosis for infants with monogenic conditions in as little as $19.5 \mathrm{~h}$ [6], potentially revolutionising clinical practice. ES-based rapid genomic testing specifically captures the protein-coding regions within the nuclear genome, while GS amplifies almost all of the genome, including intergenic regions and the mitochondrial genome [7, 8]. While GS provides additional genomic data, it is up to three times more costly than ES [9], and challenges in bioinformatics and the interpretation of non-coding variants currently limit its routine clinical use [9]. As laboratories overcome challenges associated with GS, this technique is likely to become the genomic test of choice [9]. 
Mitochondrial disorders often present with non-specific features that overlap with non-mitochondrial conditions, and as they may be caused by variants in both the mitochondrial and nuclear genomes, they pose a particular diagnostic challenge [2, 7]. In contrast to GS, ES does not target the mitochondrial genome. While mitochondrial DNA (mtDNA) variants can sometimes be identified by ES in off-target reads, depth of coverage is typically relatively low and too variable for this to be regarded as a clinical-grade diagnostic test $[10,11]$. As dedicated rapid mitochondrial genome sequencing is not usually performed in centres that have reported ES-based rapid genomic diagnosis programmes [12, 13], mitochondrial disorders caused by variants in the mitochondrial genome may remain undiagnosed within rapid timeframes. This report describes two critically ill neonates who underwent rapid mitochondrial genome sequencing in tandem with ES as part of an ES-based rapid genomic diagnosis programme.

\section{Subjects and methods}

\section{Clinical case descriptions}

The first patient was a female infant born at 36 weeks' gestation to healthy, non-consanguineous parents of Chinese ethnicity, following a pregnancy complicated by unexplained intrauterine fetal growth restriction. She developed pallor and lethargy on the fourth day of life, with severe anaemia and lactic acidosis on initial capillary blood gas (Table 1). Haematological evaluation of a

Table 1 Summary of presenting clinical features

\begin{tabular}{|c|c|c|c|}
\hline & Infant 1 & Infant 2 & Reference range \\
\hline Ethnicity & Chinese & Filipino & \\
\hline Consanguinity & Non-consanguineous & Non-consanguineous & \\
\hline Gestation & $36+2$ weeks & 40 weeks & \\
\hline Birth weight & $1810 \mathrm{~g}$ & $3118 \mathrm{~g}$ & \\
\hline Centile & 2 nd & 17 th & \\
\hline$Z$-score & -2.14 & -0.97 & \\
\hline Age at presentation & 4 days & $4 \mathrm{~h}$ & \\
\hline Presenting clinical feature & Pallor, lethargy & Tachypnoea & \\
\hline \multicolumn{4}{|l|}{ Laboratory values at presentation } \\
\hline $\mathrm{pH}$ & 7.15 & 7.11 & $7.32-7.46$ \\
\hline Base deficit & -21 & -12 & -5 to 5 \\
\hline Lactate $(\mathrm{mmol} / \mathrm{L})$ & 13 & 11 & $1.0-1.8$ \\
\hline Haemoglobin (g/L) & 57 & 91 & $125-205$ \\
\hline White cell count $\left(\times 10^{9} / \mathrm{L}\right)$ & 7.4 & 7 & $6-26$ \\
\hline Neutrophil count $\left(\times 10^{9} / \mathrm{L}\right)$ & 3.1 & 1.9 & $3.2-13.8$ \\
\hline Platelets $\left(\times 10^{9} / \mathrm{L}\right)$ & 309 & 325 & $150-450$ \\
\hline Nijmegen score-total & 4 & 2 & \\
\hline \multicolumn{4}{|l|}{ Clinical } \\
\hline Muscular & 0 & 0 & \\
\hline Neurological & 0 & 0 & \\
\hline Multisystem & 2 (GI disease, cardiomyopathy) & 0 & \\
\hline \multicolumn{4}{|l|}{ Metabolic $^{\mathrm{a}}$} \\
\hline Elevated lactate & 2 & 2 & \\
\hline Elevated alanine & 0 & 0 & \\
\hline Krebs' cycle intermediates & 0 & 0 & \\
\hline Ethyl malonic and methyl malonic acid & 0 & 0 & \\
\hline 3 methyl glutaconic acid & 0 & 0 & \\
\hline CSF lactate, alanine & 0 & 0 & \\
\hline Imaging/other ${ }^{\mathrm{b}}$ & 0 & 0 & \\
\hline
\end{tabular}

${ }^{a}$ Lactate was the only metabolic test requested at the time of recruitment

${ }^{b}$ MRI brain was not requested for either infant 
pre-transfusion blood film was non-diagnostic. The infant underwent laparotomy for necrotising enterocolitis with resection of necrotic segments of the ileum and colon. She required repeated transfusions for persistent anaemia, thrombocytopaenia and coagulopathy, and developed multiple organ dysfunction with conjugated hyperbilirubinemia, proximal renal tubular disease and myocardial hypertrophy.

The second patient was a male infant born at 40 weeks' gestation to healthy, non-consanguineous parents of Filipino ethnicity, following an uncomplicated pregnancy and delivery. He developed respiratory distress in the fourth hour of life, with anaemia and lactic acidosis on initial capillary blood gas (Table 1). Haematological evaluation of a pre-transfusion blood film was non-diagnostic. He also required multiple transfusions for persistent anaemia, thrombocytopaenia and coagulopathy.

Initial investigations for both infants were nondiagnostic. With a broad differential diagnosis, they were enrolled in an ES-based rapid genomic testing programme, the Australian Genomics Acute Care study. Clinical suspicion of mitochondrial pathology, based on modified Nijmegen criteria [14, 15] (Table 1) and the presence of two of the three classical features of Pearson syndrome (elevated lactate, transfusion-dependent anaemia, pancreatic insufficiency) [16], prompted rapid mitochondrial genome sequencing in tandem with ES. Further dedicated metabolic investigations were purposely deferred pending the results of rapid genomic testing.

\section{Clinical samples, DNA extraction and quality assessment}

Collection of an appropriate clinical specimen for DNA extraction and analysis from the first infant was complicated by frequent blood product transfusions, including repeated plasma transfusions that may contain a higher concentration of cell-free and plasma-derived donor DNA and therefore pose a theoretical risk of DNA contamination. The newborn screening dried blood spot (Guthrie card), which yields sufficient DNA for genomic studies [17], was collected prior to the first transfusion and was considered the least compromised clinical specimen. DNA was extracted from this clinical specimen using a customised in-house method modified from the Gentra Puregene Blood kit (Qiagen, Düsseldorf, Germany) for both nuclear (nDNA) and mtDNA analysis. Parallel DNA extraction was performed on post-transfusion buccal brush and peripheral blood specimens using the NucleoBond CB 20 kit (Macherey-Nagel Düren, Germany) and the QIAamp DNA blood mini kit (Qiagen), respectively. DNA extraction from a post-transfusion peripheral blood specimen collected from the second infant, and from peripheral blood specimens collected from all parents, was performed using the QIAamp DNA blood mini kit (Qiagen). DNA concentration was measured using the Invitrogen Qubit dsDNA HS Assay kit (Thermo Fisher Scientific, Waltham, MA, USA), and quality metrics for all samples were assessed using the Agilent Tape Station Genomic DNA ScreenTape (Agilent Technologies, Santa Clara, CA, USA).

\section{Genomic sequencing, data analysis and interpretation}

Trio ES of the probands and parents, and mitochondrial genome sequencing of the probands and maternal samples, were performed in tandem at clinically accredited laboratory (Victorian Clinical Genetics Services, Melbourne, Australia). ES was performed by massively parallel sequencing using SureSelect QXT CRE exome capture (Agilent Technologies) on a NextSeq 500 sequencer (Illumina, San Diego, CA, USA), with a targeted mean coverage of $100 \times$ with a minimum of $90 \%$ of bases sequenced to at least $15 \times$. Variants were characterised using the DRAGEN ${ }^{\mathrm{TM}}$ (Dynamic Read Analysis for GENomics) Bio-IT Platform (Illumina) to generate annotated variant calls within the target region (coding exons \pm 2 base pairs), via alignment to the reference genome (GRCh37).

The whole mitochondrial genome of $16.5 \mathrm{~kb}$ was amplified in a single long-range PCR, followed by library preparation using the Illumina Nextera XT DNA kit (Illumina). Sequencing was performed on a MiSeq (Illumina) using v 2 chemistry. Data were processed using the on-board MiSeq Reporter for variant calling and a custom in-house analysis pipeline for annotation and indel calling. This assay detects single nucleotide variants with heteroplasmy $>3 \%$ and large indels $>1 \mathrm{~kb}$ with heteroplasmy $>30 \%$.

Variant prioritisation by a multidisciplinary team, including clinical geneticists and medical genomics scientists, was phenotype-driven as previously described [4, 18]. Variants were assessed in accordance with the American College of Medical Genetics and Genomics (ACMG) variant classification criteria [19] by the curating medical genomics scientists, with variant classifications reviewed by the multidisciplinary team prior to reporting. Variants were submitted to ClinVar (www.ncbi.nlm.nih.gov/clinvar) (Submission ID: SUB5819049).

\section{Results}

\section{Genomic testing}

Sufficient DNA for ES and mitochondrial genome sequencing was extracted from all samples, with sufficient quality 
metrics. No diagnostic variants were identified on examination of nuclear exome data in either proband. A large mtDNA deletion was identified in both infants (Fig. 1), encompassing 4.9 kb (NC_012920.1:m.8480_13440del) in the first infant (detected in all tested samples), and $5.1 \mathrm{~kb}$ (NC_012920.1:m.8350_13450del) in the second infant (peripheral blood). The degree of heteroplasmy could not be conclusively determined due to potential amplification bias, however both variants appeared near-homoplasmic (Fig. 1). Further heteroplasmy testing was not undertaken as this was unlikely to influence clinical management. No mtDNA deletions or variants were detected in either maternal sample. Both deletions were classified as pathogenic, diagnostic of Pearson syndrome (MIM 557000). The time from laboratory receipt of clinical samples to issue of diagnostic exome and mtDNA reports was $73 \mathrm{~h} 55 \mathrm{~min}$, and $54 \mathrm{~h}$ 25 min, respectively.

\section{Clinical follow-up}

The first infant received a diagnosis of Pearson syndrome on day 18 of life. She had persistent transfusion-dependent pancytopaenia with episodes of febrile neutropaenia and ongoing multiple organ dysfunction. With a guarded prognosis, her family elected to redirect care towards palliation and symptomatic management. The infant was discharged home at age 8 weeks and died at age 11 weeks.

The second infant received a diagnosis of Pearson syndrome on day 15 of life. He required periodic blood product transfusions and developed exocrine pancreatic insufficiency. Following discharge home at 8 weeks, he subsequently developed treatment-resistant fungal sepsis with superimposed bacterial sepsis. His family elected to redirect care towards palliation and symptomatic management, and he died at age 16 weeks.

\section{Discussion}

Distinguishing genetic conditions from more common non-genetic conditions such as sepsis in acutely unwell infants is complicated by non-specific and overlapping clinical features, and incomplete phenotypic manifestations of genetic conditions at birth [1]. Diagnosis of mitochondrial conditions caused by mtDNA variants is further complicated by degrees of heteroplasmy, which may influence disease severity, and evolution of clinical phenotypes [7, 16]. Consequently, in the pre-genomic testing era as few as $8 \%$ of individuals with mitochondrial disorders received a diagnosis in the first year of life [20], and most individuals with mitochondrial disease received a preceding incorrect diagnosis, consulting as many as eight clinicians before mitochondrial pathology was correctly identified [21].

As rapid genomic testing emerges as a valuable diagnostic tool in paediatrics, earlier diagnosis of critically ill infants and children has the potential to target therapy, clarify prognosis, avoid unnecessary or invasive
A
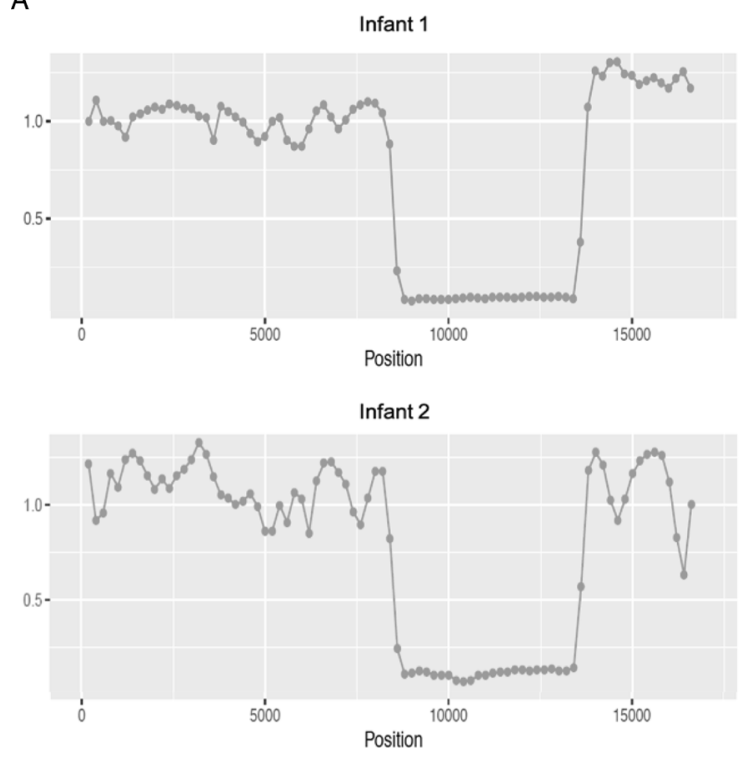

Fig. 1 Rapid mitochondrial genome sequencing results. a mtDNA sequencing binned coverage plot demonstrating the decrease in coverage relative to average coverage of a normal reference set, corresponding with the deleted segments from both infants. b Schematic

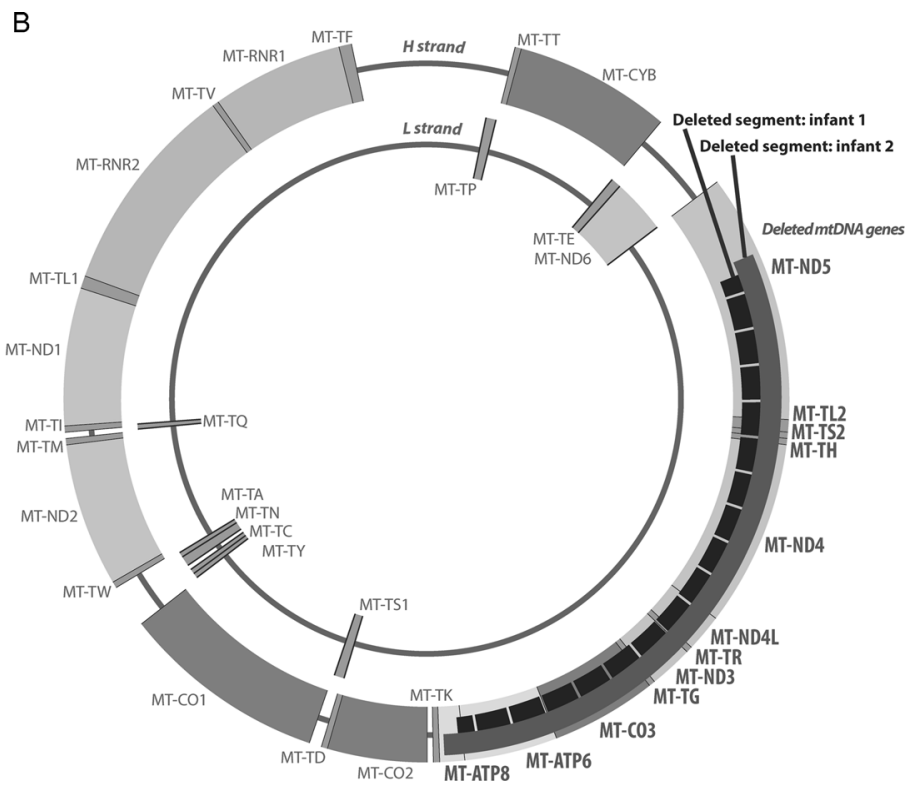

representation of circular mtDNA showing the deleted segments in the black broken bar for infant 1 , and the solid grey bar for infant 2 , with the deleted mtDNA genes shown in grey bold 
investigations and interventions, and reduce health costs [12, 13, 22-26]. GS presents challenges for widespread clinical implementation, including greater bioinformatic burden and uncertainty with interpretation of non-coding region variants [9], and has not yet surpassed ES as the preferred technique for clinical genomic testing in most centres [9]. ES detects variants in the coding regions of mitochondrial genes in the nuclear genome [27], however sufficient coverage is generally not achieved for the mitochondrial genome, which therefore must be specifically and separately tested in cases of suspected mitochondrial pathology [27]. While ES may detect single nucleotide variants in mtDNA [28, 29], the impact of nuclear mitochondrial DNA segments (NUMTs), particularly when using whole blood, has not been fully resolved [30], and ES does not provide clinical-grade assessment of mtDNA deletions and duplications $[10,11]$.

Although the differential diagnosis for acutely unwell infants and children with non-specific clinical features often includes mitochondrial disorders, rapid mitochondrial genome analysis is not routinely available as a complementary assay in ES-based rapid genomic diagnosis programmes. This is reflected in the lack of diagnosis of mitochondrial disorders caused by mtDNA variants in rapid genomic diagnosis programmes. Of 264 published cases of critically ill newborns and children who underwent rapid genomic testing [12, 13, 22-26], 116 individuals received a genomic diagnosis, including 50 of 103 individuals who underwent ES, and 66 of 161 individuals who underwent GS. Of these, only a single individual, who underwent GS, was diagnosed with a mitochondrial disorder caused by a mtDNA variant, specifically a single nucleotide variant [23]. To our knowledge, the two patients presented in this report are the first diagnoses of a mitochondrial condition caused by a large mtDNA deletion as part of a rapid genomic diagnosis programme. The low rate of detection of mitochondrial disorders may reflect an overreliance on Nijmegen scores in infants early in their illness, when rapid genomic testing is initiated, as clinical features of mitochondrial disorders, such as Pearson syndrome, evolve over time and infants may not manifest sufficient features to prompt mitochondrial testing without a high index of clinical suspicion. In these cases, Nijmegen scores $[4,2]$ indicated possible mitochondrial disease, which in the presence of elevated lactates and transfusion-dependent anaemia raised sufficient clinical suspicion to initiate mitochondrial genome sequencing in tandem with ES.

Clinical access to rapid genomic testing is presently limited to a small number of centres, using either ES or GS $[1,12,22,25,26]$. With clear economic and clinical benefits of a molecular diagnosis early in the illness trajectory [5, 12], we anticipate increasing adoption of rapid genomic diagnosis programmes. Due to the challenges associated with GS [9], ES may be preferentially adopted by many centres during the early integration of rapid genomic testing into mainstream clinical practice. To reliably facilitate diagnosis of mitochondrial conditions caused by mtDNA variants in ES-based rapid genomic diagnosis programmes, such programmes need to consider incorporating rapid mitochondrial genome sequencing for individuals with suspected mitochondrial pathology. Based on the extrapolation of cost savings observed with rapid genomic testing $[5,12]$, we expect that early diagnosis of mitochondrial disorders will be cost-effective, particularly if mitochondrial genome sequencing is performed selectively when mitochondrial pathology is clinically suspected. Challenges for implementing rapid mitochondrial genome sequencing into ES-based rapid genomic testing include the complexities of differential heteroplasmy levels between tissues, such that sequencing of mtDNA extracted from peripheral blood may fail to detect tissue-specific mtDNA variants in clinically affected tissues, such as muscle, heart and kidney [11]. Invasive tissue sampling is unlikely to be incorporated into first-line rapid testing protocols, but rather reserved for second-line testing if genomic testing on peripheral blood does not yield a diagnosis and clinical suspicion of mitochondrial pathology remains high.

In conclusion, rapid genomic testing is an attractive diagnostic tool in acute paediatric and neonatal settings, with well-established benefits of the early diagnosis of monogenic conditions. We have demonstrated the feasibility and utility of incorporating rapid mitochondrial genome sequencing in tandem with ES in infants whose differential diagnosis includes mitochondrial disorders. Centres offering ES-based rapid genomic diagnosis programmes should consider incorporating rapid mitochondrial genome sequencing in individuals with suspected mitochondrial pathology.

Acknowledgements The authors would like to acknowledge the valued and important contributions from clinical and laboratory personnel involved in the care and diagnosis of both patients, in particular Alison Compton, Ksenija Nesic, Dean Phelan, Sarah-Jane Pantaleo, Shannon Cowie, Vanessa Siva Kumar, Zeliha Hizli, Katherine Rose, Michaela Cormack, Arvind Sehgal, Hannah Brogan, Mohan Bagur Krishnamurthy, Radhakrishnan Kottayam, Lyndon Gallacher, Joy Lee, Sarah Donoghue, Sharmila Kiss, Elizabeth Wheeler and Leah Hickey.

Funding This research was funded by the Australian National Health and Medical Research Council Targeted Call for Research (GNT1113531) and the RCH Foundation (2017-906). The research conducted at the Murdoch Children's Research Institute was supported by the Victorian Government's Operational Infrastructure Support Program.

\section{Compliance with ethical standards}

Conflict of interest The authors declare that they have no conflict of interest. 
Ethics The Australian Genomics Acute Care study has Human Research Ethics Committee approval (HREC/16/MH251). Following genetic counselling, parents provided informed written consent for rapid genomic testing.

Publisher's note: Springer Nature remains neutral with regard to jurisdictional claims in published maps and institutional affiliations.

\section{References}

1. Friedman JM, Bombard Y, Cornel MC, Fernandez CV, Junker AK, Plon SE, et al. Genome-wide sequencing in acutely ill infants: genomic medicine's critical application? Genet Med. 2019;21:498-504.

2. Honzik T, Tesarova M, Magner M, Mayr J, Jesina P, Vesela K, et al. Neonatal onset of mitochondrial disorders in 129 patients: clinical and laboratory characteristics and a new approach to diagnosis. J Inherit Metab Dis. 2012;35:749-59.

3. Powis Z, Farwell Hagman KD, Speare V, Cain T, Blanco K, Mowlavi LS, et al. Exome sequencing in neonates: diagnostic rates, characteristics, and time to diagnosis. Genet Med. 2018;20:1468-71.

4. Stark Z, Tan TY, Chong B, Brett GR, Yap P, Walsh M, et al. A prospective evaluation of whole-exome sequencing as a first-tier molecular test in infants with suspected monogenic disorders. Genet Med. 2016;18:1090-6.

5. Stark Z, Schofield D, Martyn M, Rynehart L, Shrestha R, Alam K, et al. Does genomic sequencing early in the diagnostic trajectory make a difference? a follow-up study of clinical outcomes and cost-effectiveness. Genet Med. 2019;21:173-80.

6. Guinness World Records. 8th ed. Guinness World Records Limited, London: UK; 2018.

7. Raymond FL, Horvath R, Chinnery PF. First-line genomic diagnosis of mitochondrial disorders. Nat Rev Genet. 2018; 19:399-400.

8. Bowdin SC. The clinical utility of next-generation sequencing in the neonatal intensive care unit. CMAJ. 2016;188:786-7.

9. Wright CF, FitzPatrick DR, Firth HV. Paediatric genomics: diagnosing rare disease in children. Nat Rev Genet. 2018;19:325.

10. Miller DK, Menezes MJ, Simons C, Riley LG, Cooper ST, Grimmond SM, et al. Rapid identification of a novel complex I MT-ND3 m.10134C >A mutation in a Leigh syndrome patient. PLoS ONE 2014;9:e104879.

11. Taylor RW, Thorburn DR. Laboratory investigation of mitochondrial diseases. In: Chinnery PF, Keogh MJ, editors. Clinical Mitochondrial Medicine. Cambridge, UK: Cambridge University Press; 2018. p. 39-51.

12. Stark Z, Lunke S, Brett GR, Tan NB, Stapleton R, Kumble S, et al. Meeting the challenges of implementing rapid genomic testing in acute pediatric care. Genet Med. 2018;20:1554-63.

13. Meng L, Pammi M, Saronwala A, Magoulas P, Ghazi AR, Vetrini $\mathrm{F}$, et al. Use of exome sequencing for infants in intensive care units: ascertainment of severe single-gene disorders and effect on medical management. JAMA Pediatr. 2017;171: e173438.

14. Wolf NI, Smeitink JA. Mitochondrial disorders: a proposal for consensus diagnostic criteria in infants and children. Neurology. 2002;59:1402-5.
15. Witters P, Saada A, Honzik T, Tesarova M, Kleinle S, Horvath R, et al. Revisiting mitochondrial diagnostic criteria in the new era of genomics. Genet Med. 2018;20:444-51.

16. Farruggia P, Di Marco F, Dufour C. Pearson syndrome. Expert Rev Hematol. 2018;11:239-46.

17. Bassaganyas L, Freedman G, Vaka D, Wan E, Lao R, Chen F, et al. Whole exome and whole genome sequencing with dried blood spot DNA without whole genome amplification. Hum Mutat. 2018;39:167-71.

18. Stark Z, Dashnow H, Lunke S, Tan TY, Yeung A, Sadedin S, et al. A clinically driven variant prioritization framework outperforms purely computational approaches for the diagnostic analysis of singleton WES data. Eur J Hum Genet. 2017;25:1268-72.

19. Richards S, Aziz N, Bale S, Bick D, Das S, Gastier-Foster J, et al. Standards and guidelines for the interpretation of sequence variants: a joint consensus recommendation of the American College of Medical Genetics and Genomics and the Association for Molecular Pathology. Genet Med. 2015;17:405-24.

20. Sanderson S, Green A, Preece MA, Burton H. The incidence of inherited metabolic disorders in the West Midlands, UK. Arch Dis Child. 2006;91:896-9.

21. Grier J, Hirano M, Karaa A, Shepard E, Thompson JLP. Diagnostic odyssey of patients with mitochondrial disease: results of a survey. Neurol Genet. 2018;4:e230.

22. Farnaes L, Hildreth A, Sweeney NM, Clark MM, Chowdhury S, Nahas S, et al. Rapid whole-genome sequencing decreases infant morbidity and cost of hospitalization. NPJ Genom Med. 2018;3:10.

23. Willig LK, Petrikin JE, Smith LD, Saunders CJ, Thiffault I, Miller NA, et al. Whole-genome sequencing for identification of Mendelian disorders in critically ill infants: a retrospective analysis of diagnostic and clinical findings. Lancet Respir Med. 2015;3:377-87.

24. Petrikin JE, Cakici JA, Clark MM, Willig LK, Sweeney NM, Farrow EG, et al. The NSIGHT1-randomized controlled trial: rapid whole-genome sequencing for accelerated etiologic diagnosis in critically ill infants. NPJ Genom Med. 2018;3:6.

25. van Diemen CC, Kerstjens-Frederikse WS, Bergman KA, de Koning TJ, Sikkema-Raddatz B, van der Velde JK, et al. Rapid targeted genomics in critically Ill newborns. Pediatrics. 2017;140: e20162854.

26. Mestek-Boukhibar L, Clement E, Jones WD, Drury S, Ocaka L, Gagunashvili A, et al. Rapid paediatric sequencing (RaPS): comprehensive real-life workflow for rapid diagnosis of critically ill children. J Med Genet. 2018;55:721-8.

27. Kohda M, Tokuzawa Y, Kishita Y, Nyuzuki H, Moriyama Y, Mizuno Y, et al. A comprehensive genomic analysis reveals the genetic landscape of mitochondrial respiratory chain complex deficiencies. PLoS Genet. 2016;12:e1005679.

28. Wagner M, Berutti R, Lorenz-Depiereux B, Graf E, Eckstein G, Mayr JA, et al. Mitochondrial DNA mutation analysis from exome sequencing - a more holistic approach in diagnostics of suspected mitochondrial disease. J Inherit Metab Dis. 2019. [Epub ahead of print].

29. Bergant G, Maver A, Lovrecic L, Cuturilo G, Hodzic A, Peterlin B. Comprehensive use of extended exome analysis improves diagnostic yield in rare disease: a retrospective survey in 1,059 cases. Genet Med. 2018;20:303-12.

30. Ring JD, Sturk-Andreaggi K, Alyse Peck M, Marshall C. Bioinformatic removal of NUMT-associated variants in mitotiling nextgeneration sequencing data from whole blood samples. Electrophoresis. 2018;39:2785-97. 